\title{
ACADEMY OF SCIENCES OF THE CZECH REPUBLIC MATHEMATICAL INSTITUTE
}

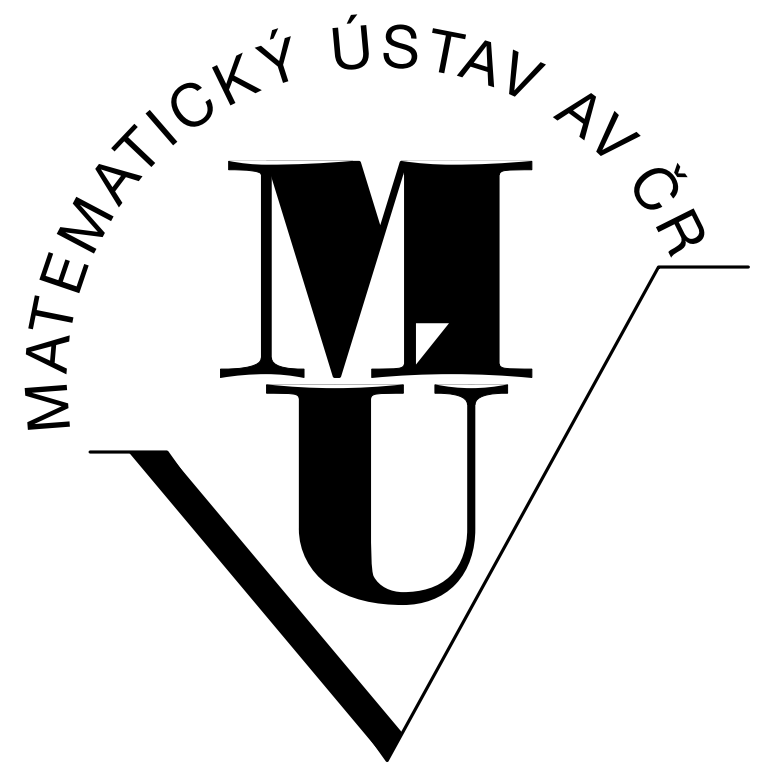

ON THE MOTION OF SEVERAL RIGID BODIES IN A VISCOUS MULTIPOLAR FLUID

Eduard Feireisl and Šárka Nečasová

(preprint)

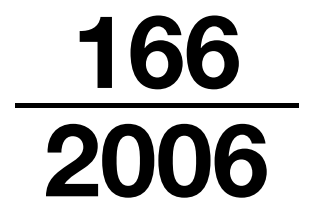




\title{
On the motion of several rigid bodies in a viscous multipolar fluid
}

\author{
Eduard Feireis/* \\ Šárka Nečasová* \\ Mathematical Institute of the Academy of Sciences of the Czech Republic \\ Žitná 25, 11567 Praha 1, Czech Republic
}

\section{Introduction}

The mathematical theory of viscous multipolar fluids, based on the general ideas of Green and Rivlin [8], was proposed by Nečas and Šilhavý [17] (see also Nečas et al. [15], [16] for relevant existence theory) in order to develop a general framework for studying viscous fluids and to present a suitable alternative to the boundary layer theory (see Bellout et al. [1]). The theory is compatible with the basic principles of thermodynamics as well as with the principle of material frame indifference. The present paper is concerned with the mathematical description of the motion of one or several rigid bodies immersed in a viscous multipolar fluid. The principal and very natural idea behind the analysis presented below is the fact that the dissipation of mechanical energy, being much stronger than for classical newtonian fluids, yields better estimates on the gradient of the velocity field, in particular, the streamlines are well-defined, which seems crucial for this class of problems partially formulated in terms of the Lagrangean coordinate system.

\subsection{Bodies and motions}

From the mathematical viewpoint, a rigid body can be identified with a connected compact subset $\bar{S}$ of the Euclidean space $R^{3}$, the motion of which is represented as a mapping $\eta:(0, T) \times R^{3} \rightarrow R^{3}$, where

$$
\eta(t, \cdot): R^{3} \rightarrow R^{3} \text { is an isometry }
$$

for any time $t \in(0, T)$. Throughout the whole text, we adopt the Eulerian (spatial) description of motion, where the coordinate system is attached to a fixed region of the physical space currently occupied by the fluid. The place $\mathbf{x}$ and the time $t \in(0, T)$ play the role of independent variables.

As the mappings $\eta(t, \cdot)$ are isometries, we can write

$$
\eta(t, \mathbf{x})=\mathbf{X}_{g}(t)+\mathbb{O}(t)\left(\mathbf{x}-\mathbf{X}_{g}(0)\right)
$$

*The work of E.F. supported by Grant IAA100190606 of GA ASCR, the work of Š.N. supported by Grant IAA100190505 of GA ASCR, both being part of the general research programme of the Academy of Sciences of the Czech Republic, Institutional Research Plan AV0Z10190503 
where $\mathbf{X}_{g}$ stands for the position of the center of mass at a time $t$, and $\mathbb{O}(t)$ is a matrix satisfying $\mathbb{O}^{T} \mathbb{O}=\mathbb{I}$. Assuming the motion to be absolutely continuous with respect to time, we introduce

$$
\frac{\mathrm{d}}{\mathrm{d} t} \mathbf{X}_{g}=\mathbf{U}_{g} \text { - the translation velocity, }
$$

and

$$
\frac{\mathrm{d}}{\mathrm{d} t} \mathbb{O}(t) \mathbb{O}^{T}(t)=\mathbb{Q}(t) \text { - the angular velocity. }
$$

Accordingly, the solid velocity in the Eulerian coordinate system can be written in the form

$$
\mathbf{u}^{S}(t, \mathbf{x})=\frac{\partial \eta}{\partial t}\left(t, \eta^{-1}(t, \mathbf{x})\right)=\mathbf{U}_{g}(t)+\mathbb{Q}(t)\left(\mathbf{x}-\mathbf{X}_{g}(t)\right),
$$

where $\mathbf{X}_{g}$ is determined through (1.2).

The total force $\mathbf{F}^{S}$ acting on the body $\bar{S}$ can be written as a sum of the body force and the contact force, more specifically,

$$
\mathbf{F}^{S}(t)=\int_{\bar{S}(t)} \varrho^{S} \mathbf{g}^{S} \mathrm{~d} \mathbf{x}+\int_{\partial \bar{S}(t)} \mathbb{T} \mathbf{n} \mathrm{d} \sigma
$$

where $\mathbb{T}$ denotes the Cauchy stress, $\mathbf{g}^{S}$ is the specific body force, and

$$
\bar{S}(t)=\eta(t, \bar{S}) .
$$

Thus Newton's second law gives rise to

$$
m \frac{\mathrm{d}}{\mathrm{d} t} \mathbf{U}_{g}(t)=\frac{\mathrm{d}}{\mathrm{d} t} \int_{\bar{S}(t)} \varrho^{S} \mathbf{u}^{S} \mathrm{~d} \mathbf{x}=\int_{\bar{S}(t)} \varrho^{S} \mathbf{g}^{S} \mathrm{~d} \mathbf{x}+\int_{\partial \bar{S}(t)} \mathbb{T} \mathbf{n} \mathrm{d} \sigma,
$$

where $m$ denotes the total mass of the body.

On the other hand, as the angular velocity $\mathbb{Q}$ is skew-symmetric, there exists a vector $\omega$ such that

$$
\mathbb{Q}(t)\left(\mathbf{x}-\mathbf{X}_{g}\right)=\omega(t) \times\left(\mathbf{x}-\mathbf{X}_{g}\right) .
$$

The balance of moment of momentum reads

$$
\begin{gathered}
\frac{\mathrm{d}}{\mathrm{d} t}(\mathbb{J} \omega)=\frac{\mathrm{d}}{\mathrm{d} t} \int_{\bar{S}(t)} \varrho^{S}\left(\mathbf{x}-\mathbf{X}_{g}\right) \times \mathbf{u}^{S} \mathrm{~d} \mathbf{x}= \\
\int_{\partial \bar{S}(t)}\left(\mathbf{x}-\mathbf{X}_{g}\right) \times \mathbb{T} \mathbf{n} \mathrm{d} \sigma+\int_{\bar{S}(t)} \varrho^{S}\left(\mathbf{x}-\mathbf{X}_{g}\right) \times \mathbf{g}^{S} \mathrm{~d} \mathbf{x},
\end{gathered}
$$

where $\mathbb{J}$ is the inertial tensor that can be identified through formula

$$
\mathbb{J} \mathbf{a} \cdot \mathbf{b}=\int_{\bar{S}(t)} \varrho^{S}\left(\mathbf{a} \times\left(\mathbf{x}-\mathbf{X}_{g}\right)\right) \cdot\left(\mathbf{b} \times\left(\mathbf{x}-\mathbf{X}_{g}\right)\right) \mathrm{d} \mathbf{x} .
$$

Equations (1.4), (1.5) determine completely the motion of the rigid body initially occupying the spatial domain $\bar{S}$. 


\subsection{The fluid motion}

In what follows, we shall assume that the state of the fluid is completely determined by its density $\varrho^{f}$ and the velocity $\mathbf{u}^{f}$ satisfying the standard mass and momentum balance equations:

$$
\begin{gathered}
\partial \varrho^{f}+\operatorname{div}_{x}\left(\varrho^{f} \mathbf{u}^{f}\right)=0, \\
\partial_{t}\left(\varrho^{f} \mathbf{u}^{f}\right)+\operatorname{div}_{x}\left(\varrho^{f} \mathbf{u}^{f} \otimes \mathbf{u}^{f}\right)+\nabla_{x} p=\operatorname{div}_{x} \mathbb{S}+\varrho^{f} \mathbf{g}^{f},
\end{gathered}
$$

where the symbol $p$ denotes the pressure, $\mathbf{g}^{f}$ is the specific body force, and $\mathbb{S}$ denotes the viscous stress tensor related to the total stress through Stokes' law:

$$
\mathbb{T}=\mathbb{S}-p \mathbb{I} .
$$

In the present paper, the effect of the temperature on the motion will be ignored. On the other hand, we consider a general compressible fluid so that the state equation relates the pressure to the fluid density through an empirical formula

$$
p=p\left(\varrho^{f}\right) .
$$

\subsection{Viscosity}

The heart of the theory of multipolar fluids lies in a particular choice of constitutive equations relating the fluid stress expressed through $\mathbb{S}$ to the symmetric component of the velocity gradient. Very roughly indeed, one can say that, in contrast to the classical theory of newtonian fluids, the stress tensor depends on higher order gradients of the velocity field. This piece of information is sufficient in order to obtain a priori estimates yielding, in particular, strong compactness of the density $\varrho$.

Following the seminal paper by Nečas and Šilhavý [17] we assume the viscous stress tensor $\mathbb{S}$ to be given as

$$
\mathbb{S}[\mathbf{u}]=\sum_{n=0}^{k-1}(-1)^{n} \Delta^{n}\left[\mu_{n}\left(\nabla_{x} \mathbf{u}+\nabla_{x} \mathbf{u}^{t}-\frac{2}{3} \operatorname{div}_{x} \mathbf{u} \mathbb{I}\right)+\zeta_{n} \operatorname{div}_{x} \mathbf{u I}\right], \mathbf{u}=\mathbf{u}^{f}
$$

where $\mu_{n}, \zeta_{n}$ are (constant) viscosity coefficients, and the symbol $\Delta$ stands for the standard Laplace operator. Accordingly, one can speak about a $k$-polar fluid, the classical newtonian fluids being identified as monopolar with $k=1$.

As expected, the presence of higher-order viscosities provides very strong a priori estimates on the velocity gradient, in particular, the streamlines are well defined allowing for the Lagrangean description of motion. Note that the theory of multipolar fluids requires additional "higher order" stresses to be introduced in the energy equation in order to comply with the second law of thermodynamics (see Nečas and Šilhavý [17]).

\subsection{Boundary conditions}

A proper choice of the boundary conditions represents one of the most delicate issues of the present theory. We adopt the hypothesis of complete adherence of the fluid to the boundaries of rigid objects yielding the full-stick boundary conditions

$$
D_{x}^{j} \mathbf{u}^{f}=D_{x}^{j} \mathbf{u}^{S}, j=0, \ldots, k-1 \text {, on } \partial \bar{S}(t),
$$


and

$$
D_{x}^{j} \mathbf{u}^{f}=0, j=0, \ldots, k-1, \text { on } \partial \Omega
$$

provided the flow is confined to a fixed spatial domain $\Omega \subset R^{3}$.

Here, the symbol $D_{x}^{j}$ denotes the vector of all spatial derivatives of order $j$, and conditions (1.11), (1.12) are different from those considered in [17], the latter being of "Neumann-type". Clearly, the boundary conditions depend on the physical properties of a given fluid and as such must be determined by experiments. In the present setting, the flowing rigid objects are supposed to be "sticky", in particular, they may be thought of as integral parts of the surrounding fluid of extremely high viscosity.

\subsection{Global-in-time solutions and collisions of rigid objects}

The motion of one or several rigid bodies in a viscous fluid has been a topic of numerous theoretical studies. Desjardins and Esteban [3] establish the existence of local-in-time solutions for incompressible newtonian fluids, where "local" is to be understood "up to the first collision of two rigid objects" if $\Omega \subset R^{2}$, or "up to the blowup of the velocity gradient in a certain Sobolev norm" in the case $\Omega \subset R^{3}$. Similar methods are proposed in [4] in order to study both compressible and incompressible case. Similar existence results "up to the first collision" were obtained by Conca et al. [2], Gunzburger et al. [9], Hoffmann and Starovoitov [12], among others.

As we have seen, the problem of existence or rather non-existence of collisions is important not only because of its practical implications, but also from the purely theoretical point of view. To the best of our knowledge, this issue remains largely open even for a two-dimensional physical domain $\Omega$. In the 2-D case, however, there is a remarkable result by San Martin et al. [18] stating, in particular, that possible collisions, if any, must be "smooth", that means, with zero relative velocities. Another strong evidence of absence of collisions in the 2-D geometry is provided independently by Hesla [10] and Hillairet [11]. These authors show, roughly speaking, that newtonian viscosity is strong enough to prevent collisions provided the rigid objects are discs. As already pointed out, the question is completely open for a linearly viscous fluid in the realistic situation $\Omega \subset R^{3}$ (for partial results see Starovoitov [20]).

The main objective of the present paper is to estalish the existence of globalin-time solutions for problem $(1.1-1.12)$ provided $k \geq 3$. In particular, we show that collisions cannot occur in a finite time unless they were already included in the initial data. The paper is organized as follows. In Section 2, we introduce a variational (weak) formulation of the problem in the spirit of Galdi [7], Hoffmann and Starovoitov [12], Serre [19]. The main results concerning global-in-time solutions are stated in Section 3. Suitable approximate solutions are constructed in Section 4 by means of a scheme similar to that used in [6]. In particular, the method of construction is based on the idea of San Martin et al. [18], where the rigid objects are approximated by a fluid of large viscosity. Such an approach is of course intimately related to our choice of the boundary conditions specified through (1.11), (1.12). In Section 5 , we derive suitable uniform estimates on the sequence of approximate solutions based on strong dissipation of the kinetic energy for multipolar fluids. In particular, the velocity field is uniformly Lipschitz continuous which facilitates the subsequent analysis considerably. Using the uniform energy estimates, we pass to the limit in the sequence of approximate solutions in order to obtain a suitable variational solution of the original problem (see Section 6). 


\section{Variational formulation}

Similarly to a major part of the reference material mentioned above, our approach is based on a suitable variational formulation of the problem. In what follows, we shall assume that $\Omega \subset R^{3}$ is a bounded domain with smooth $\left(C^{\infty}\right)$ boundary.

\subsection{Kinematics of the rigid bodies}

The reference position $S^{i}, i=1, \ldots, m$, of the $\mathrm{i}$-th rigid body is a bounded domain in $R^{3}$ with smooth boundary. The motions are described through affine isometries $\eta^{i}(t, \cdot): R^{3} \rightarrow R^{3}, i=1, \ldots, m$, that are absolutely continuous as functions of the time $t \in[0, T]$. Furthermore, we set

$$
S^{i}(t)=\eta^{i}\left(t, S^{i}\right), i=1, \ldots, m,
$$

and

$$
Q^{S}=\left\{(t, \mathbf{x}) \mid t \in(0, T), \mathbf{x} \in \cup_{i=1}^{m} S^{i}(t)\right\} .
$$

\subsection{Conservation of mass}

The solid densities $\varrho^{S^{i}}$ as well as the fluid density $\varrho^{f}$ can be extended to be zero outside $S^{i}(t)$, and the fluid region $Q^{f}=((0, T) \times \Omega) \backslash \bar{Q}^{S}$, respectively. In a similar way, we introduce a "global" velocity field $\mathbf{u}$,

$$
\mathbf{u}(t, \mathbf{x})=\left\{\begin{array}{l}
\mathbf{u}^{S^{i}} \text { for } t \in(0, T), \mathbf{x} \in \bar{S}^{i}(t), \\
\mathbf{u}^{f} \text { for } t \in(0, T), \mathbf{x} \in \Omega \backslash \cup_{i=1}^{m} \bar{S}^{i}(t), \\
0 \text { for } t \in(0, T), \mathbf{x} \in R^{3} \backslash \Omega .
\end{array}\right.
$$

The physical principle of mass conservation can be expressed through continuity equation

$$
\partial_{t} \varrho+\operatorname{div}_{x}(\varrho \mathbf{u})=0 \text { in } \mathcal{D}^{\prime}\left((0, T) \times R^{3}\right) .
$$

\subsection{Momentum equation}

For a given family of motions $\eta^{i}, i=1, \ldots, m$, we introduce the set of admissible velocity fields

$V_{\mathrm{adm}}(t)=\left\{\mathbf{w} \in C^{k}\left(R^{3} ; R^{3}\right) \mid \mathbf{w} \equiv 0\right.$ on $R^{3} \backslash \Omega, \nabla_{x} \mathbf{w}+\nabla_{x} \mathbf{w}^{t} \equiv 0$ on $\left.\cup_{i=1}^{m} \eta^{i}\left(t, S^{i}\right)\right\}$.

Following Nečas [14], we introduce a bilinear form $((\cdot, \cdot))$ associated to the stress tensor $\mathbb{S}$ given by $(1.10)$, specifically,

$$
((\mathbf{v}, \mathbf{w}))=\int_{\Omega} \mathbb{S}[\mathbf{v}]: \nabla_{x} \mathbf{w} \mathrm{d} x \text { for any } \mathbf{v}, \mathbf{w} \in \mathcal{D}\left(\Omega ; R^{3}\right) .
$$

Under the natural hypothesis

$$
\mu_{n}, \eta_{n} \geq 0, \mu_{k-1}>0, \eta_{k-1}>0
$$

it is straightforward to see that $((\cdot, \cdot))$ can be extended to a scalar product on the Sobolev space $W_{0}^{k, 2}\left(\Omega ; R^{3}\right)$ defined as a completion of the set of compactly supported 
smooth functions with respect to the norm

$$
\|\mathbf{v}\|_{W_{0}^{k, 2}\left(\Omega ; R^{3}\right)}^{2}=\sum_{n=0}^{k} \int_{\Omega}\left|D_{x}^{n} \mathbf{v}\right|^{2} \mathrm{~d} x
$$

(see Nečas [14]).

Assuming continuity of the stresses on the boundaries of rigid objets, we can reformulate equations (1.4), (1.5), (1.7) in terms of $\varrho$ and $\mathbf{u}$ as integral identity

$$
\begin{gathered}
\int_{0}^{T} \int_{R^{3}}\left(\varrho \mathbf{u} \cdot \partial_{t} \mathbf{w}+\varrho \mathbf{u} \otimes \mathbf{u}: \nabla_{x} \mathbf{w}+p \operatorname{div}_{x} \mathbf{w}\right) \mathrm{d} \mathbf{x} \mathrm{d} t= \\
\int_{0}^{T}((\mathbf{u}, \mathbf{w})) \mathrm{d} t-\int_{0}^{T} \int_{R^{3}} \varrho \mathbf{g} \cdot \mathbf{w} \mathrm{d} \mathbf{x} \mathrm{d} t-\int_{R^{3}} \varrho_{0} \mathbf{u}_{0} \cdot \mathbf{w}(0) \mathrm{d} \mathbf{x}
\end{gathered}
$$

to be satisfied for any test function

$$
\mathbf{w} \in C^{1}\left([0, T] ; C^{k}\left(R^{3}\right)\right), \mathbf{w}(T)=0, \mathbf{w}(t) \in V_{\mathrm{adm}}(t) \text { for any } t \in[0, T] .
$$

Note that (2.5) includes the initial conditions

$$
(\varrho \mathbf{u})(0, \cdot)=\varrho_{0} \mathbf{u}_{0}
$$

\subsection{Compatibility of the "global" velocity with rigid motions}

We shall say that a velocity field $\mathbf{u}$ is compatible with the family of rigid motions $\eta^{i}$, $i=1, \ldots, m$ provided

$$
\mathbf{u}(t, \mathbf{x})=\mathbf{u}^{S^{i}}(t, \mathbf{x})=\frac{\partial \eta^{i}}{\partial t}\left(t,\left(\eta^{i}\right)^{-1}(t, \mathbf{x})\right) \text { for all } \mathbf{x} \in \eta^{i}\left(t, S^{i}\right), i=1, \ldots, m .
$$

Relation (2.8) is to be satisfied for any $t \in[0, T]$.

\subsection{Energy inequality}

The velocity field associated to a multipolar fluid is expected regular because of the strong kinetic energy dissipation in the high velocity gradient regime. Indeed taking (formally) a test function $\mathbf{w}=-\psi(t) \mathbf{u}$, in (2.5) we obtain energy inequality

$$
E\left(t_{2}\right)+\int_{t_{1}}^{t_{2}}((\mathbf{u}, \mathbf{u})) \mathrm{d} t \leq E\left(t_{1}\right)+\int_{t_{1}}^{t_{2}} \int_{\Omega} \varrho \mathbf{g} \cdot \mathbf{u} \mathrm{d} x
$$

for any $0 \leq t_{1} \leq t_{2} \leq T$, with

$$
E(t)=\int_{\Omega}\left(\frac{1}{2} \varrho|\mathbf{u}|^{2}+\varrho P(\varrho)\right) \mathrm{d} x,
$$

where $P$ is related to the pressure $p$ through

$$
P(\varrho)=\varrho \int_{1}^{\varrho} \frac{p(z)}{z^{2}} \mathrm{~d} z .
$$




\subsection{Weak (variational) solutions}

Having collected all the preliminary material, we are in a position to introduce the concept of weak solution to our problem referred to hereafter as problem $(\mathbf{P})$.

\section{Definition 2.1}

Let the initial distribution of the density and the velocity field be determined through given functions $\varrho_{0}$ and $\mathbf{u}_{0}$, respectively; the initial position of the rigid bodies being $S^{i} \subset \Omega, i=1, \ldots, m$. We say that a family $\varrho, \mathbf{u}, \eta^{i}, i=1, \ldots, m$, represent a variational solution of problem $(\mathbf{P})$ on a time interval $(0, T)$ provided the following conditions are satisfied:

- The density $\varrho$ is a non-negative bounded function, the velocity field $\mathbf{u}$ belongs to the space $L^{\infty}\left(0, T ; L^{2}\left(\Omega ; R^{3}\right)\right) \cap L^{2}\left(0, T ; W_{0}^{k, 2}\left(\Omega ; R^{3}\right)\right)$, and they satisfy energy inequality (2.9) for $t_{1}=0$ and a.a. $t_{2} \in(0, T)$, with

$$
E(0)=E_{0}=\int_{\Omega}\left(\frac{1}{2} \varrho_{0}\left|\mathbf{u}_{0}\right|^{2}+\varrho_{0} P\left(\varrho_{0}\right)\right) \mathrm{d} x .
$$

- We have $\varrho \in C\left([0, T] ; L^{1}(\Omega)\right), \varrho(0)=\varrho_{0}$, and continuity equation (2.1) holds on $(0, T) \times R^{3}$ provided $\varrho$ and $\mathbf{u}$ were extended to be zero outside $\Omega$.

- Momentum equation (the integral identity) (2.5) holds for any admissible test function $\mathbf{w}$ satisfying (2.6).

- The mappings $\eta^{i}, i=1, \ldots, m$ are affine isometries of $R^{3}$ compatible with the velocity field $\mathbf{u}$ in the sense of (2.8).

\section{Global existence - main results}

Our main goal is to prove the following existence result.

Theorem 3.1 Let $\Omega \subset R^{3}, S^{i} \subset R^{3}, i=1, \ldots, m$ be a family of bounded domains with boundaries of class $C^{\infty}$ such that

$$
\bar{S}^{i} \cap \bar{S}^{j}=\emptyset \text { for } i \neq j, \bar{S}^{i} \subset \Omega \text { for } i=1, \ldots, m .
$$

Furthermore, assume that $\varrho_{0}$ is a measurable function such that

$$
0<\underline{\varrho} \leq \varrho_{0}(x) \leq \bar{\varrho} \text { for a.a. } x \in \Omega,
$$

and that

$$
\mathbf{u}_{0} \in W_{0}^{k, 2}\left(\Omega ; R^{3}\right) \cap V_{\text {adm }}(0) \text { for a certain } k \geq 3 .
$$

Finally, let $\mathbf{g} \in L^{\infty}\left(\Omega ; R^{3}\right)$, and $p \in C[0, \infty)$ - a non-decreasing function be given.

Then problem $(\mathbf{P})$ admits a variational solution $\varrho, \mathbf{u}, \eta^{i}, i=1, \ldots, m$, in the sense of Definition 2.1 on an arbitrary time interval $(0, T)$. Moreover, we have

$$
\bar{S}^{i}(t) \cap \bar{S}^{j}(t)=\emptyset \text { for } i \neq j, \bar{S}^{i}(t) \subset \Omega \text { for } i=1, \ldots, m
$$

for any $t \in[0, T]$, that means, the motion is smooth without any collision of two or several rigid objects in a finite time. 
As already pointed out several times, the main ingredient of the proof are strong dissipation estimates resulting from energy inequality (2.9). Since $k \geq 3$, the velocity field $\mathbf{u}$ is a priori bounded in the space $L^{2}\left(0, T ; C_{0}^{1}(\bar{\Omega})\right)$, in particular, the streamlines (characteristics) can be identified with the unique solution of the system

$$
\frac{\mathrm{d}}{\mathrm{d} t} \mathbf{X}(t, \mathbf{x})=\mathbf{u}(t, \mathbf{X}(t, \mathbf{x})), t>0, \mathbf{X}(0, \mathbf{x})=\mathbf{x} .
$$

Accordingly, the (unique) weak solution $\varrho$ of $(2.1)$ satisfying $\varrho(0)=\varrho_{0}$ is given by formula

$$
\varrho(t, \mathbf{X}(t, \mathbf{x}))=\varrho_{0}(x) \exp \left(-\int_{0}^{t} \operatorname{div}_{x} \mathbf{u}(s, \mathbf{X}(s, \mathbf{x})) \mathrm{d} s\right), \mathbf{x} \in R^{3} .
$$

Note that, in the absence of collisions, one can deduce from (2.5) that

$$
\partial_{t}(\varrho \mathbf{u}) \in L^{2}\left(0, T ; W^{-k, 2}\left(\Omega ; R^{3}\right)\right),
$$

which, combined with (2.9), gives rise to

$$
\mathbf{u} \in C\left([0, T] ; L^{2}\left(\Omega ; R^{3}\right)\right) .
$$

In particular, formula (3.6) makes sense for any weak solution in the sense of Definition 2.1 .

The rest of the paper is devoted to the proof of Theorem 3.1.

\section{Approximate problems}

Let $\left\{\mathbf{v}_{n}\right\}_{n=1}^{\infty} \subset \mathcal{D}\left(\Omega ; R^{3}\right)$ be a basis of the Hilbert space $W_{0}^{k, 2}\left(\Omega ; R^{3}\right)$. Following San Martin et al. [18] we introduce the approximate problem $(\mathbf{P})_{n, \varepsilon}$ :

$$
\begin{gathered}
\int_{0}^{T} \int_{R^{3}}\left(\varrho_{n, \varepsilon} \mathbf{u}_{n, \varepsilon} \cdot \partial_{t} \mathbf{w}+\varrho_{n, \varepsilon} \mathbf{u}_{n, \varepsilon} \otimes \mathbf{u}_{n, \varepsilon}: \nabla_{x} \mathbf{w}+p\left(\varrho_{n, \varepsilon}\right) \operatorname{div}_{x} \mathbf{w}\right) \mathrm{d} \mathbf{x} \mathrm{d} t= \\
\int_{0}^{T} \int_{\Omega} M_{\varepsilon}\left(\chi_{n, \varepsilon}\right)\left[\nabla_{x} \mathbf{u}_{n, \varepsilon}+\nabla_{x}^{t} \mathbf{u}_{n, \varepsilon}\right]:\left[\nabla_{x} \mathbf{w}+\nabla_{x}^{t} \mathbf{w}\right] \mathrm{d} x \mathrm{~d} t+ \\
\int_{0}^{T}\left(\left(\mathbf{u}_{n, \varepsilon}, \mathbf{w}\right)\right) \mathrm{d} t-\int_{0}^{T} \int_{R^{3}} \varrho_{n, \varepsilon} \mathbf{g} \cdot \mathbf{w} \mathrm{d} \mathbf{x} \mathrm{d} t-\int_{R^{3}} \varrho_{0} \mathbf{u}_{0} \cdot \mathbf{w}(0) \mathrm{d} \mathbf{x}
\end{gathered}
$$

to be satisfied for any test function

$$
\mathbf{w} \in C^{1}\left([0, T] ; X_{n}\right), \mathbf{w}(T)=0, X_{n}=\operatorname{span}\left\{\mathbf{v}_{1}, \ldots, \mathbf{v}_{n}\right\} .
$$

Here, $\varrho_{n, \varepsilon}$ is determined through formula

$$
\varrho_{n, \varepsilon}\left(t, \mathbf{X}_{n, \varepsilon}(t, \mathbf{x})\right)=\varrho_{0}(x) \exp \left(-\int_{0}^{t} \operatorname{div}_{x} \mathbf{u}_{n, \varepsilon}\left(s, \mathbf{X}_{n, \varepsilon}(s, \mathbf{x})\right) \mathrm{d} s\right), \mathbf{x} \in R^{3}
$$

while

$$
\chi_{n, \varepsilon}\left(t, \mathbf{X}_{n, \varepsilon}(t, \mathbf{x})\right)=\chi_{0, \varepsilon}(\mathbf{x}) \geq 0
$$

where

$$
\frac{\mathrm{d}}{\mathrm{d} t} \mathbf{X}_{n, \varepsilon}(t, \mathbf{x})=\mathbf{u}_{n, \varepsilon}\left(t, \mathbf{X}_{n, \varepsilon}(t, \mathbf{x})\right), t>0, \mathbf{X}_{n, \varepsilon}(0, \mathbf{x})=\mathbf{x}
$$


Note that $\chi_{n, \varepsilon}$ is the unique distributional solution of the equation

$$
\partial_{t} \chi_{n, \varepsilon}+\mathbf{u}_{n, \varepsilon} \cdot \nabla_{x} \chi_{n, \varepsilon}=0, \chi_{n, \varepsilon}(0)=\chi_{0, \varepsilon}
$$

(cf. DiPerna and Lions [5]).

The functions $M_{\varepsilon}$ belong to the class $C^{1}[0, \infty) \cap B C[0, \infty)$ for any fixed $\varepsilon>0$.

Problem $(\mathbf{P})_{n, \varepsilon}$ can be solved by means of the standard fixed-point argument used in [13, Section 2], the presence of the additional viscosity coefficient $M_{\varepsilon}$ requiring only minor modifications.

\section{$5 \quad$ Uniform estimates}

Similarly to Section 2.5, one can show that the approximate solutions $\varrho_{n, \varepsilon}, \mathbf{u}_{n, \varepsilon}$ satisfy energy equality

$$
\begin{gathered}
E_{n, \varepsilon}\left(t_{2}\right)+\int_{t_{1}}^{t_{2}} \int_{\Omega} M_{\varepsilon}\left(\chi_{n, \varepsilon}\right)\left|\nabla_{x} \mathbf{u}_{n, \varepsilon}+\nabla_{x}^{t} \mathbf{u}_{n, \varepsilon}\right|^{2} \mathrm{~d} x \mathrm{~d} t+\int_{t_{1}}^{t_{2}}\left(\left(\mathbf{u}_{n, \varepsilon}, \mathbf{u}_{n, \varepsilon}\right)\right) \mathrm{d} t=(5.1) \\
E_{n, \varepsilon}\left(t_{1}\right)+\int_{t_{1}}^{t_{2}} \int_{\Omega} \varrho_{n, \varepsilon} \mathbf{g} \cdot \mathbf{u}_{n, \varepsilon} \mathrm{d} x
\end{gathered}
$$

for any $0 \leq t_{1} \leq t_{2} \leq T$, with

$$
\begin{aligned}
& E_{n, \varepsilon}(t)=\int_{\Omega}\left(\frac{1}{2} \varrho_{n, \varepsilon}\left|\mathbf{u}_{n, \varepsilon}\right|^{2}+\varrho_{n, \varepsilon} P\left(\varrho_{n, \varepsilon}\right)\right) \mathrm{d} x, \\
& E_{n, \varepsilon}(0)=E_{0}=\int_{\Omega}\left(\frac{1}{2} \varrho_{0}\left|\mathbf{u}_{0}\right|^{2}+\varrho_{0} P\left(\varrho_{0}\right)\right) \mathrm{d} x .
\end{aligned}
$$

Under the hypotheses of Theorem 3.1, it is a routine matter to check that (5.1 5.3) give rise to uniform estimates:

$$
\begin{gathered}
\left\{\mathbf{u}_{n, \varepsilon}\right\}_{n, \varepsilon} \text { bounded in } L^{2}\left(0, T ; W_{0}^{k, 2}\left(\Omega ; R^{3}\right)\right) \\
\underline{\varrho} \exp \left(-T\left\|\operatorname{div}_{x} \mathbf{u}_{n, \varepsilon}\right\|_{L^{1}\left(0, T ; L^{\infty}(\Omega)\right)}\right) \leq \varrho_{n, \varepsilon}(t, \mathbf{x}) \leq \bar{\varrho} \exp \left(T\left\|\operatorname{div}_{x} \mathbf{u}_{n, \varepsilon}\right\|_{L^{1}\left(0, T ; L^{\infty}(\Omega)\right)}\right)
\end{gathered}
$$

for a.a. $t \in(0, T), \mathbf{x} \in \Omega$, and

$$
\left\{\mathbf{u}_{n, \varepsilon}\right\}_{n, \varepsilon} \text { bounded in } L^{\infty}\left(0, T ; L^{2}\left(\Omega ; R^{3}\right)\right) .
$$

In addition,

$$
\int_{0}^{T} \int_{\Omega} M_{\varepsilon}\left(\chi_{n, \varepsilon}\right)\left|\nabla_{x} \mathbf{u}_{n, \varepsilon}+\nabla_{x}^{t} \mathbf{u}_{n, \varepsilon}\right|^{2} \mathrm{~d} x \mathrm{~d} t \leq \text { const }
$$

where the bound is uniform with respect to $n, \varepsilon$. 


\section{Convergence}

\subsection{The limit $n \rightarrow \infty$}

With $\varepsilon>0$ fixed, our aim is to let $n \rightarrow \infty$ in the family of approximate solutions $\varrho_{n, \varepsilon}, \mathbf{u}_{n, \varepsilon}$ constructed in Section 4. To begin with, estimates (5.4), (5.6) yield

$$
\mathbf{u}_{n, \varepsilon} \rightarrow \mathbf{u}_{\varepsilon} \text { weakly in } L^{2}\left(0, T ; W_{0}^{k, 2}\left(\Omega ; R^{3}\right)\right) \text { and weakly-(*) in } L^{\infty}\left(0, T ; L^{2}\left(\Omega ; R^{3}\right)\right)
$$

for $n \rightarrow \infty$, at least for a suitable subsequence.

Similarly, by virtue of (4.3), (5.5), we can assume that

$$
\varrho_{n, \varepsilon} \rightarrow \varrho_{\varepsilon} \text { in } C_{\text {weak }}\left([0, T] ; L^{1}(\Omega)\right) \text { and weakly-(*) in } L^{\infty}\left(0, T ; L^{\infty}(\Omega)\right) .
$$

Consequently, combining (6.1), (6.2), we conclude that

$$
\varrho_{n, \varepsilon} \mathbf{u}_{n, \varepsilon} \rightarrow \varrho_{\varepsilon} \mathbf{u}_{\varepsilon} \text { weakly-(*) in } L^{\infty}\left(0, T ; L^{2}\left(\Omega ; R^{3}\right)\right) .
$$

Moreover, it follows from (4.1) that

$\left\{t \mapsto \int_{\Omega}\left(\varrho_{n, \varepsilon} \mathbf{u}_{n, \varepsilon}\right)(t) \cdot \mathbf{v}_{n} \mathrm{~d} x\right\} \rightarrow\left\{t \mapsto \int_{\Omega}\left(\varrho_{\varepsilon} \mathbf{u}_{\varepsilon}\right)(t) \cdot \mathbf{v}_{n} \mathrm{~d} x\right\}$ in $C[0, T]$ for $n=1,2, \ldots ;$ whence

$$
\varrho_{n, \varepsilon} \mathbf{u}_{n, \varepsilon} \rightarrow \varrho_{\varepsilon} \mathbf{u}_{\varepsilon} \text { in } C_{\text {weak }}\left([0, T] ; L^{2}\left(\Omega ; R^{3}\right)\right) .
$$

Relations (6.1), (6.4) imply

$$
\varrho_{n, \varepsilon} \mathbf{u}_{n, \varepsilon} \cdot \mathbf{u}_{n, \varepsilon} \rightarrow \varrho_{\varepsilon} \mathbf{u}_{\varepsilon} \cdot \mathbf{u}_{\varepsilon} \text { weakly in } L^{2}((0, T) \times \Omega) ;
$$

therefore, in view of (4.3),

$$
\mathbf{u}_{n, \varepsilon} \rightarrow \mathbf{u}_{\varepsilon} \text { in } L^{2}\left(0, T ; L^{2}\left(\Omega ; R^{3}\right)\right) .
$$

Consequently, (6.1), (6.6) and a simple interpolation argument yield

$$
\mathbf{u}_{n, \varepsilon} \rightarrow \mathbf{u}_{\varepsilon} \text { in } L^{2}\left(0, T ; W_{0}^{k-1,2}\left(\Omega ; R^{3}\right)\right),
$$

in particular,

$$
\operatorname{div}_{x} \mathbf{u}_{n, \varepsilon} \rightarrow \operatorname{div}_{x} \mathbf{u}_{\varepsilon} \text { in } L^{2}\left(0, T ; W_{0}^{k-2,2}\left(\Omega ; R^{3}\right)\right) .
$$

Since $\varrho_{n, \varepsilon}$ satisfy continuity equation (2.1), one can deduce from (6.2), (6.8) that

$$
\varrho_{n, \varepsilon} \rightarrow \varrho_{\varepsilon} \text { in } L^{1}((0, T) \times \Omega) .
$$

In a similar way, one can show

$$
\chi_{n, \varepsilon} \rightarrow \chi_{\varepsilon} \text { in } L^{1}((0, T) \times \Omega) .
$$

Thus we have shown there are functions $\varrho_{\varepsilon}, \mathbf{u}_{\varepsilon}$ such that

$$
\begin{gathered}
\int_{0}^{T} \int_{R^{3}}\left(\varrho_{\varepsilon} \mathbf{u}_{\varepsilon} \cdot \partial_{t} \mathbf{w}+\varrho_{\varepsilon} \mathbf{u}_{\varepsilon} \otimes \mathbf{u}_{\varepsilon}: \nabla_{x} \mathbf{w}+p\left(\varrho_{\varepsilon}\right) \operatorname{div}_{x} \mathbf{w}\right) \mathrm{d} \mathbf{x} \mathrm{d} t= \\
\int_{0}^{T} \int_{\Omega} M_{\varepsilon}\left(\chi_{\varepsilon}\right)\left[\nabla_{x} \mathbf{u}_{\varepsilon}+\nabla_{x}^{t} \mathbf{u}_{\varepsilon}\right]:\left[\nabla_{x} \mathbf{w}+\nabla_{x}^{t} \mathbf{w}\right] \mathrm{d} x \mathrm{~d} t+
\end{gathered}
$$




$$
\int_{0}^{T}\left(\left(\mathbf{u}_{\varepsilon}, \mathbf{w}\right)\right) \mathrm{d} t-\int_{0}^{T} \int_{R^{3}} \varrho_{\varepsilon} \mathbf{g} \cdot \mathbf{w} \mathrm{d} \mathbf{x} \mathrm{d} t-\int_{R^{3}} \varrho_{0} \mathbf{u}_{0} \cdot \mathbf{w}(0) \mathrm{d} \mathbf{x}
$$

to be satisfied for any test function

$$
\mathbf{w} \in C^{1}\left([0, T] ; W_{0}^{k, 2}\left(\Omega, R^{3}\right)\right), \mathbf{w}(T)=0 .
$$

Furthermore, we have

$$
\varrho_{\varepsilon}\left(t, \mathbf{X}_{\varepsilon}(t, \mathbf{x})\right)=\varrho_{0}(x) \exp \left(-\int_{0}^{t} \operatorname{div}_{x} \mathbf{u}_{\varepsilon}\left(s, \mathbf{X}_{\varepsilon}(s, \mathbf{x})\right) \mathrm{d} s\right), \mathbf{x} \in R^{3}
$$

and

$$
\chi_{\varepsilon}\left(t, \mathbf{X}_{\varepsilon}(t, \mathbf{x})\right)=\chi_{0, \varepsilon}(\mathbf{x}) \geq 0
$$

where

$$
\frac{\mathrm{d}}{\mathrm{d} t} \mathbf{X}_{\varepsilon}(t, \mathbf{x})=\mathbf{u}_{\varepsilon}\left(t, \mathbf{X}_{\varepsilon}(t, \mathbf{x})\right), t>0, \mathbf{X}_{\varepsilon}(0)=\mathbf{x}
$$

In addition, the energy inequality

$$
\begin{gathered}
E_{\varepsilon}\left(t_{2}\right)+\int_{t_{1}}^{t_{2}} \int_{\Omega} M_{\varepsilon}\left(\chi_{\varepsilon}\right)\left|\nabla_{x} \mathbf{u}_{\varepsilon}+\nabla_{x}^{t} \mathbf{u}_{\varepsilon}\right|^{2} \mathrm{~d} x \mathrm{~d} t+\int_{t_{1}}^{t_{2}}\left(\left(\mathbf{u}_{\varepsilon}, \mathbf{u}_{\varepsilon}\right)\right) \mathrm{d} t \leq \\
E_{\varepsilon}\left(t_{1}\right)+\int_{t_{1}}^{t_{2}} \int_{\Omega} \varrho_{\varepsilon} \mathbf{g} \cdot \mathbf{u}_{\varepsilon} \mathrm{d} x
\end{gathered}
$$

holds for any $0 \leq t_{1} \leq T$ and a.a. $t_{2} \in\left(t_{1}, T\right)$, where

$$
E_{\varepsilon}(t)=\int_{\Omega}\left(\frac{1}{2} \varrho_{\varepsilon}\left|\mathbf{u}_{\varepsilon}\right|^{2}+\varrho_{\varepsilon} P\left(\varrho_{\varepsilon}\right)\right) \mathrm{d} x, E_{\varepsilon}(0)=E_{0}=\int_{\Omega}\left(\frac{1}{2} \varrho_{0}\left|\mathbf{u}_{0}\right|^{2}+\varrho_{0} P\left(\varrho_{0}\right)\right) \mathrm{d} x .
$$

\subsection{The limit for $\varepsilon \rightarrow 0$}

Adopting the idea of San Martin et al. [18] we take

$$
\begin{gathered}
M_{\varepsilon}(z)=\frac{1}{\varepsilon} \max \{z, 0\}, \\
\chi_{\varepsilon, 0}=\chi_{0} \in C^{1}(\bar{\Omega}), \chi_{0}(\mathbf{x})=\left\{\begin{array}{l}
0 \text { for } \mathbf{x} \in \bar{\Omega} \backslash \cup_{i=1}^{m} \bar{S}^{i}, \\
>0 \text { for } \mathbf{x} \in \cup_{i=1}^{m} S^{i} .
\end{array}\right.
\end{gathered}
$$

Our ultimate goal is to let $\varepsilon \rightarrow 0$ in $(6.11$ - 6.16) in order to recover the global-in-time solution of problem (P), the existence of which is claimed in Theorem 3.1.

To begin with, we can assume, by virtue of (6.16), that

$$
\mathbf{u}_{\varepsilon} \rightarrow \mathbf{u} \text { weakly in } L^{2}\left(0, T ; W_{0}^{k, 2}\left(\Omega ; R^{3}\right)\right)
$$

passing to a suitable subsequence as the case may be. Moreover, as $\left\{\varrho_{\varepsilon}\right\}_{\varepsilon>0}$ is bounded below away from zero in view of (6.13), we have

$$
\mathbf{u}_{\varepsilon} \rightarrow \mathbf{u} \text { weakly-(*) in } L^{\infty}\left(0, T ; L^{2}\left(\Omega ; R^{3}\right)\right) .
$$

As stated in (6.13), $\left\{\varrho_{\varepsilon}\right\}_{\varepsilon>0}$ solve (in the sense of distributions) continuity equation (2.1) supplemented with the initial datum $\varrho_{0}$; whence we have

$$
\varrho_{\varepsilon} \rightarrow \varrho \text { in, say, } C_{\text {weak }}\left([0, T] ; L^{2}(\Omega)\right),
$$




$$
\varrho_{\varepsilon} \mathbf{u}_{\varepsilon} \rightarrow \varrho \mathbf{u} \text { weakly-(*) in } L^{\infty}\left(0, T ; L^{2}\left(\Omega ; R^{3}\right)\right) .
$$

Thus we are allowed to conclude that $\varrho, \mathbf{u}$, extended to be zero outside $\Omega$, solve equation (2.1). In addition, $\varrho$ is uniquely determined by $\varrho_{0}$ and the velocity field $\mathbf{u}$, and $\varrho \in C\left([0, T] ; L^{1}(\Omega)\right)$ (cf. DiPerna and Lions $[5]$ ).

In order to identify the family of isometries $\eta^{i}, i=1, \ldots, m$, we need the following auxilliary result proved in [6, Proposition 5.1].

Lemma 6.1 Let $\mathbf{u}_{\varepsilon}=\mathbf{u}_{\varepsilon}(t, \mathbf{x})$ be a family of Caratheodory functions such that

$$
\int_{0}^{T}\left\|\mathbf{u}_{\varepsilon}(t)\right\|_{W^{1, \infty}\left(R^{3} ; R^{3}\right)}^{2} \mathrm{~d} t \leq \mathrm{const}
$$

uniformly with respect to $\varepsilon \rightarrow 0$. Let $S_{\varepsilon} \subset R^{3}$ be a family of open sets such that

$$
\mathbf{d b}\left[S_{\varepsilon}\right] \rightarrow \mathbf{d b}[S] \text { in } C_{\mathrm{loc}}\left(R^{3}\right)
$$

where $S \subset R^{3}$ is an open set and the symbol $\mathbf{d b}$ denotes the signed distance from the boundary:

$$
\mathbf{d b}[S](\mathbf{x})=\operatorname{dist}\left[\mathbf{x}, \overline{R^{3} \backslash S}\right]-\operatorname{dist}[\mathbf{x}, \bar{S}], \operatorname{dist}[\mathbf{x}, K] \equiv \min _{\mathbf{y} \in K}|\mathbf{x}-\mathbf{y}| .
$$

Denote by $\mathbf{X}_{\varepsilon}$ the unique solution of the problem

$$
\frac{\mathrm{d}}{\mathrm{d} t} \mathbf{X}_{\varepsilon}(t, \mathbf{x})=\mathbf{u}_{\varepsilon}\left(t, \mathbf{X}_{\varepsilon}(t, \mathbf{x})\right), 0<t<T, \mathbf{X}_{\varepsilon}(0, \mathbf{x})=\mathbf{x} .
$$

Then, extracting a suitable subsequence if necessary, we have

$$
\mathbf{u}_{\varepsilon} \rightarrow \mathbf{u} \text { weakly-(*) in } L^{2}\left(0, T ; W^{1, \infty}\left(R^{3} ; R^{3}\right)\right),
$$

and

$$
\mathbf{X}_{\varepsilon}(t, \cdot) \rightarrow \mathbf{X}(t, \cdot) \text { in } C_{\mathrm{loc}}\left(R^{3}\right) \text { uniformly in } t \in[0, T],
$$

where $\mathbf{X}$ solves

$$
\frac{\mathrm{d}}{\mathrm{d} t} \mathbf{X}(t, \mathbf{x})=\mathbf{u}(t, \mathbf{X}(t, \mathbf{x})), 0<t<T, \mathbf{X}(0, \mathbf{x})=\mathbf{x} .
$$

Moreover,

$$
\mathbf{d b}\left[S_{\varepsilon}(t)\right] \rightarrow \mathbf{d b}[S(t)] \text { in } C_{\mathrm{loc}}\left(R^{3}\right) \text { uniformly in } t \in[0, T],
$$

where we have ser

$$
S_{\varepsilon}(t)=\mathbf{X}_{\varepsilon}\left(t, S_{\varepsilon}\right), S(t)=\mathbf{X}(t, S) .
$$

Consider the domains $S^{i}(t), i=1, \ldots, m$ occupied by the images of the rigid bodies $S^{i}, i=1, \ldots, m$ under the flow induced by $(6.24)$ :

$$
S^{i}(t)=\mathbf{X}\left(t, S^{i}\right), i=1, \ldots, m .
$$

Since the velocity field $\mathbf{u}$ belongs to the class $L^{2}\left(0, T ; C^{1}\left(R^{3} ; R^{3}\right)\right.$, we have, in accordance with hypothesis (3.1),

$$
\bar{S}^{i}(t) \cap \bar{S}^{j}(t)=\emptyset \text { for } i \neq j, \bar{S}^{i} \subset \Omega \text { for } i=1, \ldots, m
$$


for all $t \in[0, T]$, that is to say, there is no collision of two or more "rigid" objects.

Let

$$
(t, \mathbf{x}) \in \cup_{t \in(0, T)} S^{i}(t) .
$$

In accordance with Lemma 6.1, there is a small open neighbourhood $V$ of $(t, \mathbf{x})$ such that

$$
V \subset \bar{V} \subset \cup_{t \in(0, T)} \mathbf{X}_{\varepsilon}\left(t, S^{i}\right) \text { for all } \varepsilon>0 \text { small enough, }
$$

where $\mathbf{X}_{\varepsilon}$ are determined through (6.15).

Consequently, combining energy inequality (6.16) with (6.14), (6.17), (6.18) we conclude that

$$
\nabla_{x} \mathbf{u}+\nabla_{x}^{t} \mathbf{u}=0 \text { a.a. on } V \text {. }
$$

As the point $(t, \mathbf{x})$ was arbitrary, we have

$$
\nabla_{x} \mathbf{u}+\nabla_{x}^{t} \mathbf{u}=0 \text { a.a. on } \cup_{i=1}^{m} \cup_{t \in(0, T)} S^{i}(t) .
$$

It is a routine matter to deduce from $(6.24),(6.26)$ that

$$
\mathbf{X}(t, \cdot): S^{i} \rightarrow R^{3} \text { is an isometry for any } i=1, \ldots, m \text { and any fixed } t \in[0, T] ;
$$

whence we can set

$$
\eta^{i}(t, \cdot): R^{3} \rightarrow R^{3}, \eta^{i}(t, \mathbf{x})=\mathbf{X}(t, \mathbf{x}) \text { for all } \mathbf{x} \in S^{i}, t \in[0, T], i=1, \ldots, m
$$

Clearly, the family $\eta^{i}, i=1, \ldots, m$ is compatible with the vector field $\mathbf{u}$ in the sense of Definition 2.1.

In order to complete the proof of Theorem 3.1, we have to pass to the limit for $\varepsilon \rightarrow 0$ in (6.11) to recover momentum equation (2.5). To this end, first observe that

$$
\begin{gathered}
\left\{\varrho_{\varepsilon} \mathbf{u}_{\varepsilon}\right\}_{\varepsilon>0} \text { is precompact in } C_{\text {weak }}\left(\left[t_{1}, t_{2}\right], L^{2}\left(B ; R^{3}\right)\right) \\
\text { whenever }\left(\left[t_{1}, t_{2}\right] \times \bar{B}\right) \cap \cup_{i=1}^{m} \cup_{t \in[0, T]} \bar{S}^{i}(t)=\emptyset .
\end{gathered}
$$

In particular, in accordance with (6.20),

$$
\varrho_{\varepsilon} \mathbf{u}_{\varepsilon} \otimes \mathbf{u}_{\varepsilon} \rightarrow \mathbb{Q} \text { weakly in } L^{2}\left(0, T ; L^{2}\left(\Omega ; R^{3 \times 3}\right)\right),
$$

where

$$
\mathbb{Q}=\varrho \mathbf{u} \otimes \mathbf{u} \text { on } \Omega \backslash \cup_{i=1}^{m} \cup_{t \in(0, T)} S^{i}(t) .
$$

As a byproduct of (6.30) we get

$$
\mathbf{u}_{\varepsilon} \rightarrow \mathbf{u} \text { in } L^{2}\left(\Omega \backslash \cup_{i=1}^{m} \cup_{t \in(0, T)} S^{i}(t) ; R^{3}\right) ;
$$

whence, having used the estimates on $\operatorname{div}_{x} \mathbf{u}_{\varepsilon}$ resulting from (6.16), we conclude that

$$
\operatorname{div}_{x} \mathbf{u}_{\varepsilon} \rightarrow \operatorname{div}_{x} \mathbf{u} \text { in } L^{1}((0, T) \times \Omega) .
$$

Since $\varrho_{\varepsilon}$ solve the continuity equation, relation (6.32) implies

$$
\varrho_{\varepsilon} \rightarrow \varrho \text { in } L^{1}((0, T) \times \Omega) .
$$

Using (6.29), (6.33) one can let $\varepsilon \rightarrow 0$ in (6.11) in order to recover (2.5) at least for any test function w such that

$$
\mathbf{w} \in C^{1}\left([0, T] ; C^{k}\left(R^{3}\right)\right), \mathbf{w}(T)=0, \mathbf{w}(t) \in V_{\mathrm{adm}}^{\delta}(t) \text { for any } t \in[0, T],
$$


where

$$
\begin{gathered}
V_{\mathrm{adm}}^{\delta}(t)=\left\{\mathbf{w} \in C^{k}\left(R^{3} ; R^{3}\right) \mid \mathbf{w} \equiv 0 \text { on a } \delta \text {-neighbourhood of } \overline{R^{3} \backslash \Omega},\right. \\
\left.\nabla_{x} \mathbf{w}+\nabla_{x} \mathbf{w}^{t} \equiv 0 \text { on a } \delta \text {-neighbourhood of } \cup_{i=1}^{m} \overline{\eta^{i}\left(t, S^{i}\right)}\right\}, \delta>0 .
\end{gathered}
$$

Note that

$$
\int_{0}^{T} \int_{\Omega} \varrho_{\varepsilon} \mathbf{u}_{\varepsilon} \otimes \mathbf{u}_{\varepsilon}: \nabla_{x} \mathbf{w} \mathrm{d} x \mathrm{~d} t=\frac{1}{2} \int_{0}^{T} \int_{\Omega} \varrho_{\varepsilon} \mathbf{u}_{\varepsilon} \otimes \mathbf{u}_{\varepsilon}:\left(\nabla_{x} \mathbf{w}+\nabla_{x}^{t} \mathbf{w}\right) \mathrm{d} x \mathrm{~d} t .
$$

Since there is no contact of rigid objets (see (6.25)), it is easy to extend validity of (2.5) to all test functions in the class (2.6) via density argument.

Finally, by virtue of (6.19), (6.33), energy inequality (2.9) holds for $t_{1}=0$ and a.a. $t_{2} \in(0, T)$ as required in Definition 2.1. Theorem 3.1 has been proved.

\section{References}

[1] H. Bellout, F. Bloom, and J. Nečas. Phenomenological behavior of multipolar viscous fluids. Quart. Appl. Math., 50:559-583, 1992.

[2] C. Conca, J. San Martin, and M. Tucsnak. Existence of solutions for the equations modelling the motion of a rigid body in a viscous fluid. Commun. Partial Differential Equations, 25:1019-1042, 2000.

[3] B. Desjardins and M.J. Esteban. Existence of weak solutions for the motion of rigid bodies in a viscous fluid. Arch. Rational Mech. Anal., 146:59-71, 1999.

[4] B. Desjardins and M.J. Esteban. On weak solutions for fluid-rigid structure interaction: Compressible and incompressible models. Commun. Partial Differential Equations, 25:1399-1413, 2000.

[5] R.J. DiPerna and P.-L. Lions. Ordinary differential equations, transport theory and Sobolev spaces. Invent. Math., 98:511-547, 1989.

[6] E. Feireisl. On the motion of rigid bodies in a viscous compressible fluid. Arch. Rational Mech. Anal., 167:281-308, 2003.

[7] G. P. Galdi. On the steady self-propelled motion of a body in a viscous incompressible fluid. Arch. Rat. Mech. Anal., 148:53-88, 1999.

[8] A.E. Green and R.S. Rivlin. Theories of elasticity with stress multipoles. Arch. Rational Mech. Anal., 17:85-112, 1964.

[9] M.D. Gunzburger, H. C. Lee, and A. Seregin. Global existence of weak solutions for viscous incompressible flow around a moving rigid body in three dimensions. J. Math. Fluid Mech., 2:219-266, 2000.

[10] T.I. Hesla. Collision of smooth bodies in a viscous fluid: A mathematical investigation. 2005. PhD Thesis - Minnesota.

[11] M. Hillairet. Lack of collision between solid bodies in a 2D incompressible viscous flow. 2006. Preprint - ENS Lyon. 
[12] K.-H. Hoffmann and V. N. Starovoitov. On a motion of a solid body in a viscous fluid. Two dimensional case. Adv. Math. Sci. Appl., 9:633-648, 1999.

[13] Š. Matušů-Nečasová and A. Novotný. Measure-valued solution for nonnewtonian compressible isothermal monopolar fluid. Acta Applicandae Mathematicae, 37:109-128, 1994.

[14] J. Nečas. Theory of multipolar fluids. In The mathematics of finite elements and applications, Academic Press, London, VII:233-244, 1991.

[15] J. Nečas, A. Novotný, and M. Šilhavý. Global solution to the ideal compressible heat-conductive multipolar fluid. Comment. Math. Univ. Carolinae, 30:551-564, 1989.

[16] J. Nečas, A. Novotný, and M. Šilhavý. Global solutions to the compressible isothermal multipolar fluid. J. Math. Anal. Appl., 162:223-241, 1991.

[17] J. Nečas and M. Šilhavý. Viscous multipolar fluids. Quart. Appl. Math., 49:247266, 1991.

[18] J.A. San Martin, V. Starovoitov, and M. Tucsnak. Global weak solutions for the two dimensional motion of several rigid bodies in an incompressible viscous fluid. Arch. Rational Mech. Anal., 161:93-112, 2002.

[19] D. Serre. Chute libre d'un solide dans un fluid visquex incompressible. Existence. Jap. J. Appl. Math., 4:99-110, 1987.

[20] V.N. Starovoitov. Nonuniqueness of a solution to the problem on motion of a rigid body in a viscous incompressible fluid. J. Math. Sci., 130:4893-4898, 2005. 


\section{ACADEMY OF SCIENCES \\ OF THE CZECH REPUBLIC \\ MATHEMATICAL INSTITUTE}

The preprint series was founded in 1982. Its purpose is to present manuscripts of submitted or unpublished papers and reports of members of the Mathematical Institute of the Academy of Sciences of the Czech Republic.

The authors are fully responsible for the content of the preprints.

Mail address: $\quad$ Mathematical Institute,

Academy of Sciences of the Czech Republic

Žitná 25,

CZ-115 67 Praha 1

Czech Republic

phone: +420222090711

fax: +420222211638

e-mail: mathinst@math.cas.cz

http: //www.math.cas.cz

\section{Latest preprints of the series:}

\section{6}

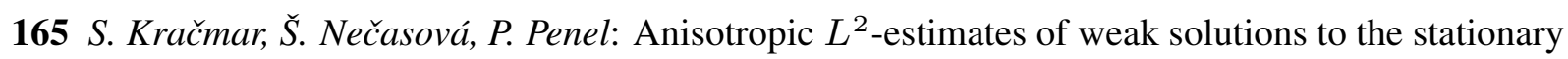
Oseen-type equations in $R^{3}$ for a rotating body

164 Patrick Penel and Ivan Straškraba: Construction of a Lyapunov functional for 1D-viscous compressible barotropic fluid equations admitting vacua

163 Martin Markl: Operads and props

2005

162 Jiřı Šremr: On the initial value problem for two-dimensional systems of linear functional differential equations with monotone operators

161 Jiř $\breve{S}$ remr: On the characteristic initial value problem for linear partial functional-differential equations of hyperbolic type

160 A. Lomtatidze, S. Mukhigulashvili, and J. Šremr: Nonnegative solutions of the characteristic initial value problem for linear partial functional-differential equations of hyperbolic type

159 Andrej Rontó: Upper bounds for the eigenvalues of compact linear operators in a preordered Banach space

158 Martin Markl: Cohomology operators and the Deligne conjecture

2004

157 J. Eisner, M. Kučera, L. Recke: A global bifurcation result for variational inequalities

156 Irena Rachůnková, Milan Tvrdý: Second order periodic problem with 8-Laplacian and impulses-part II

155 Irena Rachůnková, Milan Tvrdý: Second order periodic problem with 8-Laplacian and impulses-part I

2003

154 J. Eisner, M. Kučera, L. Recke: Direction and stability of bifurcation branches for variational inequalities

153 Irena Rachůnková, Milan Tvrdý: Periodic boundary value problems for nonlinear second order differential equations with impulses-part III

152 Pavel Krutitskii, Dagmar Medková: Neumann and Robin problem in a cracked domain with jump conditions on cracks

2002

151 Irena Rachůnková, Milan Tvrdý: Periodic boundary value problems for nonlinear second order differential equations with impulses-part II

150 Miroslav Šilhavý: An $O(n)$ invariant rank 1 convex function that is not polyconvex

149 Šárka Nečasová: Asymptotic properties of the steady fall of a body in viscous fluids 